\title{
A new approach to simulate buildings and their crucial characteristics in a comprehensive urban simulation environment
}

\author{
M. Ziegler \& T. Bednar \\ Institute of Building Construction and Technology, \\ Vienna University of Technology, Austria
}

\begin{abstract}
URBEM is an interdisciplinary cooperation between the Vienna University of Technology and Wiener Stadtwerke. One of the key elements is to find a proper simulation environment to simulate the effects of different urban development strategies (e.g. 2020, 2030, 2050) focusing on all buildings, the entire district heating and cooling grid as well as the whole gas and electricity grid within a district or even the entire city. The aim of this research is to find a validated method to create load profiles in terms of scalable density function for individual buildings and building neighborhoods regarding the heating, cooling and electricity demand with respect to certain building use cases, thermal qualities, construction year, HVAC-systems and even the influence of lifestyle aspects. This paper demonstrates a new approach how to implement buildings and their characteristics into a new developed urban simulation environment. Due to special requirements on the urban simulation environment, conventional building simulation tools are not capable in terms of time efficiency, interoperability and connectivity to deal with those particular initial parameters to run the entire simulation environment. By using density functions only the input of a certain urban development scenario, that is going to be investigated within the URBEM and the quantity of buildings, is needed to generate aggregated, location and time dissolved heating load curves. By comparing the results with a detailed simulation, the range of the relative deviations performs by $+/-1.5 \%$, which is an accurate and sufficient result for using scalable density function within the URBEM simulation environment. Keywords: urban simulation environment, load profiles, density function, energy demand validation, building simulation model, urbanization.
\end{abstract}




\section{Introduction}

Global urbanization and sustainable cities are polarizing and central catchphrases and omnipresent in the media today. Certain technologies as well as road maps towards sustainable cities already exist. Besides the implementation planners, consultants as well as political decision makers needs appropriate tools to be prepared for future challenges and to make, based on that, informed and accurate decisions.

URBEM is an interdisciplinary cooperation between the Vienna University of Technology [1] and the Wiener Stadtwerke (Vienna's biggest utility company) [2]. This comprehensive doctoral course, entitled "Urban Energy and Mobility System", covers the work of $10 \mathrm{PhD}$ candidates and 20 professors within 5 faculties at the Vienna University of Technology as well as along with many experts and decision makers of the Wiener Stadtwerke and the City Council of Vienna. The aim is to research and develop an interactive environment for analysing scenarios towards a sustainable and secure supply, affordable and liveable city by the example of the city Vienna in a holistic and interdisciplinary approach. A general visual of the URBEM [3] environment is provided in figure 1.

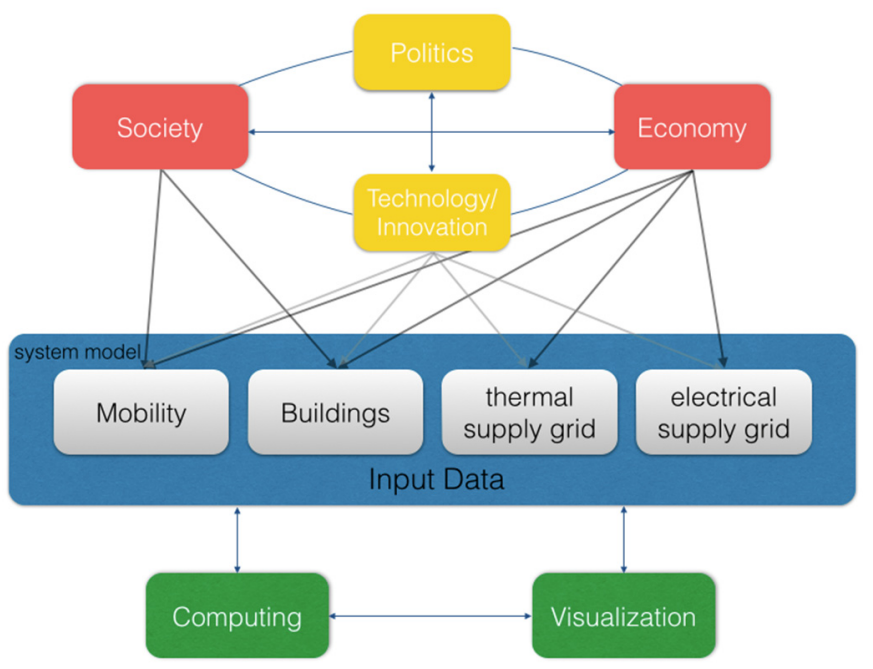

Figure 1: URBEM environment and their dependencies.

This paper addresses a new scientific approach for buildings and their utilizations to deliver both highly aggregated load profiles to the overall system as well as detail considerations at the level of local used energy technologies. By considering the overall energy supply system, the potential of several decentralized technologies gets determined with respect to an increasing share of renewables. Therefore time and location dissolved data's for buildings in terms of heating demand, cooling demand and electricity demand are crucial. 
Besides the role of buildings and their characteristics, URBEM [3] simultaneously considers the scientific disciplines mobility, the entire energy supply grid, lifestyle issues, economics as well as computing, big data and the complexity of visualization within this comprehensive simulation environment (figure 1). Each topic gets conducted by one $\mathrm{PhD}$ candidate and supervised by two Professors as well as one representative of the Wiener Stadtwerke. One key challenge within the simulation task force is to make the participating disciplines interacting to each other on every time step a year. This enables the visualization of a dynamic and interacting energy grid within an entire city depending on the investigated scenarios.

\section{Building simulation}

This comprehensive simulation environment gets newly developed. Therefore new models for the simulation and overall evaluation for buildings, thermal and gas grid as well as electrical grid needs to get implemented and make them interacting among themselves. According to Crawley et al. [4] and Maile et al. [5], state of the art building simulation programs are generally powerful and enables almost all detailed calculations to meet extensive requirements regarding the energy demand of construction physics issues. But almost all programs have one characteristics in common, they are usually used for simulating only one building in a time intensive way. The challenge for the URBEM simulation environment is to find a proper simulation tool to simulate the effects of different urban development strategies (e.g. 2020, 2030 and 2050) focusing on all buildings, the entire district heating and cooling grid as well as the hole gas and electricity grid within a district or even the entire city. Therefore different urban development scenarios, such as the annual heating or cooling demand, refurbishment rates, etc., will be used as initial parameters to run the entire URBEM simulation environment. Such individual indicators are usually insufficient to run commercial building simulation software at an accurate quality level. Too many assumptions have to be made for even starting such a tool. In order to maintain a sufficient performance and a time efficient duration within an entire urban simulation environment, the simulation efforts for single buildings supposed to be as low and as capable as possible.

To meet the above mentioned requirements a new approach has been developed. The idea is to use validated load profile in terms of density functions instead of using conventional building simulation tools. The systematic approach can be seen in figure 2 .

Figure 2 points out in clear terms the systematic process to create static load profile for different building use cases. Therefore every input parameter such as thermal standards (e.g. nearly zero energy building, passive house), lifestyle aspects, year of construction and building use case (e.g. residential building, office building) a validated density function needs to be created. The result will be a matrix of static density functions and load profiles depending on the requirement for each time step within the URBEM simulation environment. Due to all density 


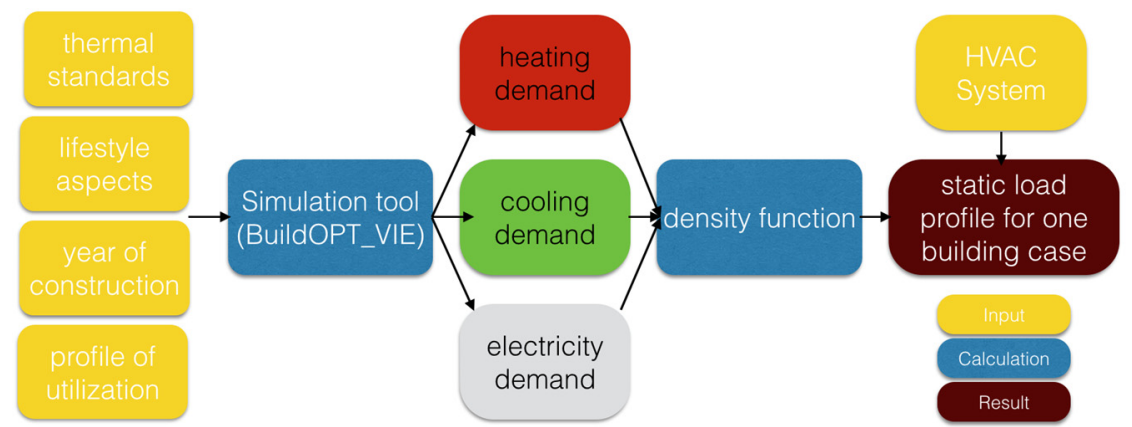

Figure 2: Systematic approach to generate a static load profile for one building use case using a validated density function.

function are scalable, only the input of the investigated URBEM scenarios and the building area within the investigated boundaries is necessary to generate time and location dissolved load profiles.

\section{Density function}

For a better understanding of the results, some crucial content of Ziegler et al. [6] will be repeated briefly in that chapter. In order to validate the density function, 4 different building models have been developed. The different developed building models can be seen in figure 3 .
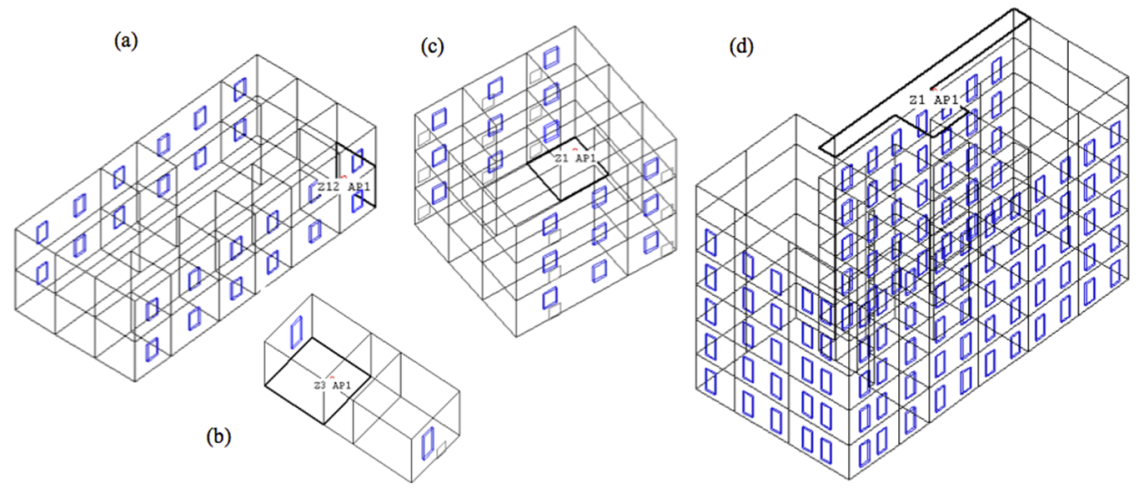

Figure 3: $\quad$ (a) 2 stories -22 zones; (b) 1 story -3 zones; (c) 3 stories -18 zones; (d) 6 stories -21 zones.

While buildings (a) and (c) represent a simple model of a fictive house, building (d) represents a typical Vienna building built before 1940, which is actually real. All building types are well represented in Vienna, that's why those models seems to be accurate case study building models for further validations within in this research. In order to achieve a fair validation, all building models have either the 
thermal quality of the current Austrian building guidelines [7] - so called BAU$\mathrm{B}$, or the thermal quality of a passive house [7] - so called NZEB.

The reference simulation to verify the correctness and level of accuracy of particular density function gets done by using the validated simulation software BuildOPT_VIE [8, 9], developed at the Research Center of Building Physics and Sound Protection at the Vienna University of Technology.

Eqn. (1) shows the calculation of the density function by using $H, n d(t, i)$ and H,nd(i).

$$
h(t, i)=\frac{H, n d(t, i)}{H, n d(i)}
$$

$h(t, i) \quad$ density function for each time step and 1 particular building model

$t \quad$ time step (h)

$i \quad$ building type/model

$H, n d(t, i) \quad$ heating demand for each time step and 1 particular building model $(\mathrm{kWh} / \mathrm{h})$

$H$,nd (i) annual heating demand for 1 particular building model (kWh/a)

According to figure 3 the following matrix, eqn. (2), for two example density function can be created.

$$
\begin{gathered}
h(t, B A U(a)) \\
h(t, \operatorname{NZEB}(a)) \\
h(t, B A U(d)) \\
h(t, \operatorname{NZEB}(d))
\end{gathered}
$$

$h(t, B A U(a)) \quad$ density function for the BAU scenario and building model (a) according to figure 3

$h(t, N Z E B(a)) \quad$ density function for the NZEB scenario and building model (a) according to figure 3

$h(t, B A U(d)) \quad$ density function for the BAU scenario and building model (d) according to figure 3

$h(t, N Z E B(d)) \quad$ density function for the NZEB scenario and building model (d) according to figure 3

In order to calculate $\mathrm{H}$,nd $(\mathrm{t}, \mathrm{i})$ by using a density function for each time step instead of a conventional simulation model, eqn. (3) will be used.

$$
h, n d(t, i)=h(t, i) \times f(i) \times A(i)
$$

$h, n d(t, i) \quad$ heating demand for each time step and particular building type using a density function $(\mathrm{kWh} / \mathrm{h})$

$h(t, i) \quad$ density function for each time step of a particular building model $f(i) \quad$ scenario objective for the specific annual heating demand $\left(\mathrm{kWh} / \mathrm{m}^{2} \mathrm{a}\right)$

A(i) floor area $\left(\mathrm{m}^{2}\right)$ 


\section{Results}

All upcoming figures [6] are basically dealing with the transformation from a BAU-B to a NZEB building and the other way round to show example results to make the approach of this paper clear. Both continuous lines (blue and green) are representing the results from the simulation with BuildOPT_VIE. Both dotted lines are representing the results using a density function. In case of building (d), a density function based on the simulation results for a BAU-B standard was used to generate the load profile for a NZEB standard (green dotted) and one density function based on the simulation results for a NZEB standard was used to generate the load profile for a BAU-B standard (blue dotted). The results show a well accurate correlation (see dashed lines) between each dotted line to simulation results.

The results for the relative deviations are in the range of $-1.07 \%$ and $+1.07 \%$ (December). While figure 4 shows only the results for one variable parameter (thermal quality; BAU-B to NZEB), figure 5 deals with the results by transforming from building (a) to building (d) and varying the thermal qualities, which equals to two variable parameters.

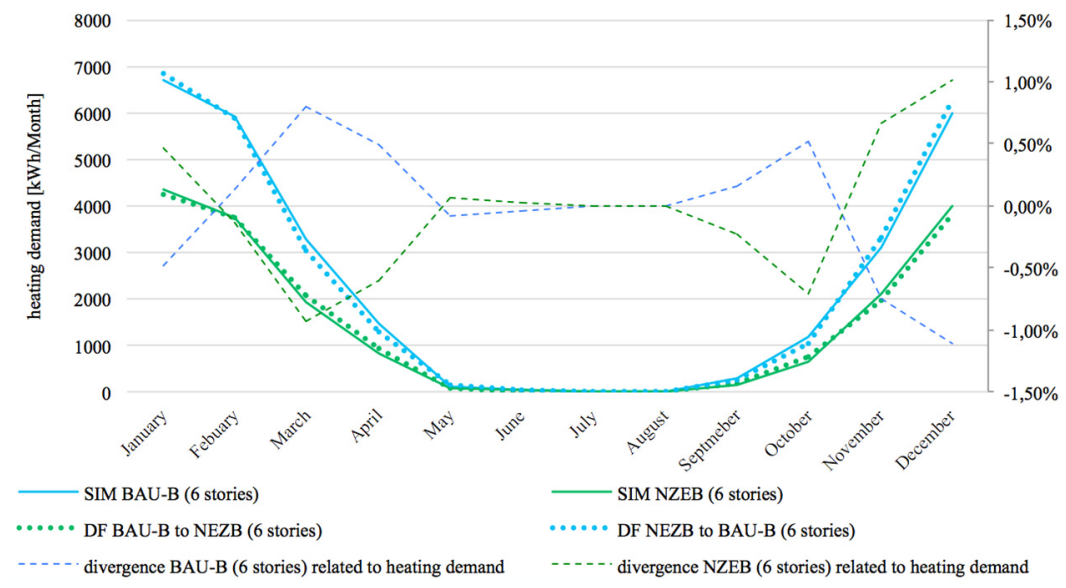

Figure 4: Monthly validation by using a density function created building (d).

The results of figure 5 are similar to figure 4, even the load profile is slightly underestimated. In this particular case the results for the relative deviations are in the range of $-1.5 \%$ (December) and $+1.2 \%$ (October). Figure 6 is showing the results for three variable parameters.

BUC1 building use case 1

BUC2 building use case 2

BUC1 and BUC2 describes different office occupations. In addition to the results of figure 5, figure 6 is showing if the building use case gets varied additionally. The relative deviations performs in a range of $+3 \%$ (November) and $-2.5 \%$ (February). 


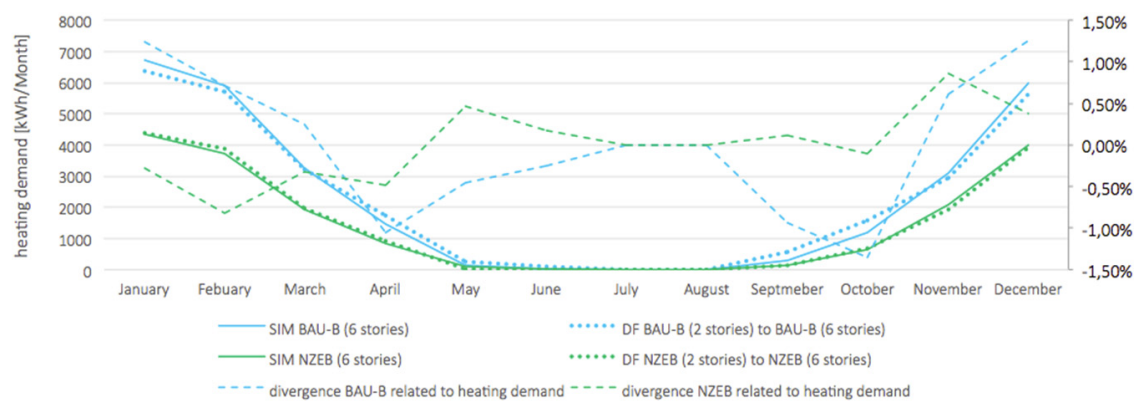

Figure 5: Monthly validation by using a density function created by building (a) to create a load profile for building $(\mathrm{d})$.

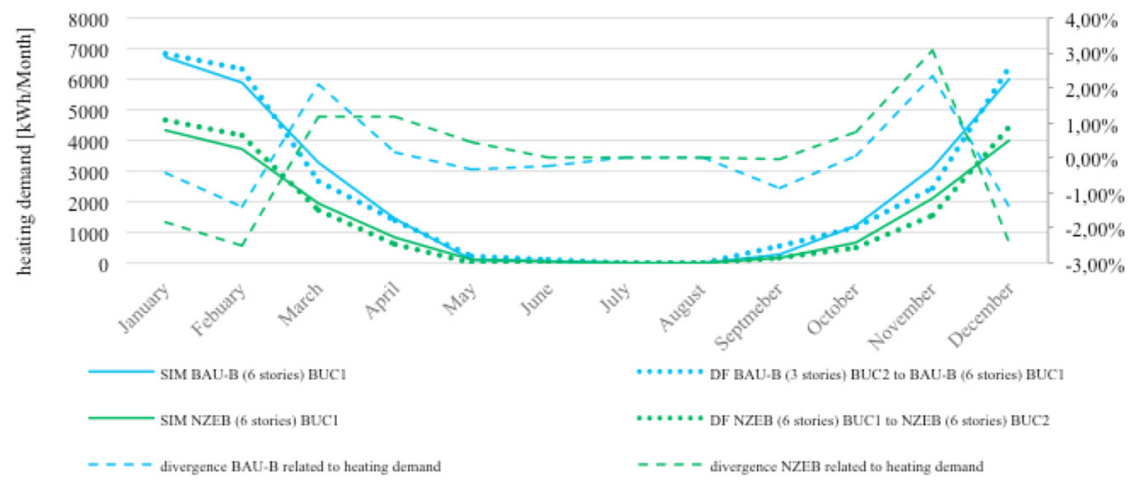

Figure 6: Monthly validation by using a density function created on BUC2/ building (c) to create a load profile for BUC1 on building (d).

By analysing the results, it can be seen in table 1 that the relative deviation is increasing by an increasing number of variable parameters.

Table 1: Summary of the results sorted by the number of variables.

\begin{tabular}{|c|c|}
\hline Amount of variables & Relative deviation \\
\hline 1 (figure 4$)$ & $+1.07 \% \ldots-1.07 \%$ \\
\hline 2 (figure 5$)$ & $+1.2 \% \ldots-1.5 \%$ \\
\hline 3 (figure 6$)$ & $+3 \% \ldots-2.5 \%$ \\
\hline
\end{tabular}

In order to keep the matrix for different density function clear and easy to use within the URBEM simulation environment, the number of different density functions supposed to be as low as possible with respect to the qualities of the results. Therefore not more than 2 variable parameters gets determined by using one density function within the URBEM simulation environment. 
Even the validation for a daily load profile is not done yet, figure 7 shows a sneak peak of the current status within this research for a daily validation.

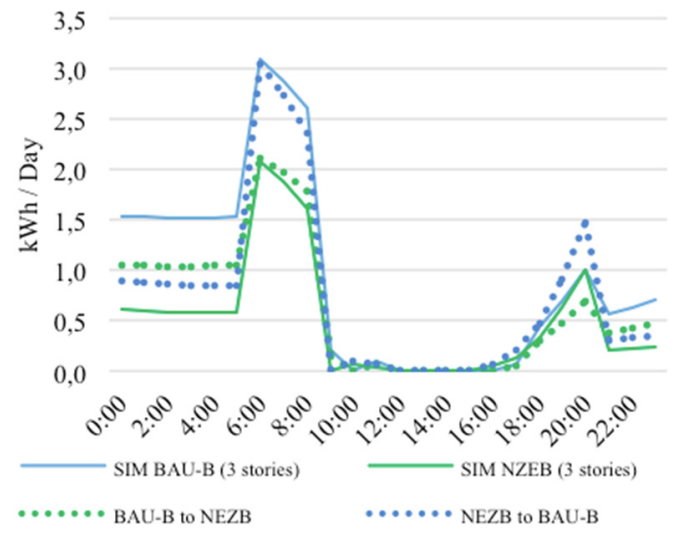

Figure 7: One day load profile with 2 variable parameters (1st March).

Within the operating hours of this office building the results are well accurate. Even the influence of internal gains due to the office occupation gets covered by the density function. During the evening hours the deviation is increasing due to a lower simultaneity factor at this particular occupation.

\section{URBEM simulation environment}

The comprehensive URBEM simulation environment enables a time and location dissolved simulation in terms of energy supply, energy generation, energy distribution and mobility. Therefore different scenarios for the building stock such as annual heating demand for individual buildings, refurbishment rates, etc., are the initial parameters to run the entire simulation environment. Depending on the investigated scenarios, a density functions gets distributed to every building within the considered boundaries. This could be either a building neighbourhood, a district or even the entire city.

Due to the interdisciplinary nature and holistic approach, $10 \mathrm{PhD}$ candidates make all tasks simultaneously within the URBEM [3]. The workflow can be divided into four main work packages (figure 8). The first work package represents the economic approach. This part is responsible for all economic boundaries and scenario driven conditions. The results of this work package are all initial parameters for the simulation environment, which represents the second work package. Example results of the simulation work package are time and location dissolved energy flows. Starting with the heating, cooling and electricity demand of individual buildings to detailed energy flow calculations for the entire energy distribution. The third work package deals with all visualization issues. All results get visualized for a better understanding and can be seen as the basis for decision makers and other stakeholder. The fourth work package runs simultaneously to the 
first three packages and is mainly responsible for all computing issues. All main results, sub results, simulation sequences, simulation interoperability, data structure, data security and outcome for further research gets controlled by the computing sector within the URBEM [3]

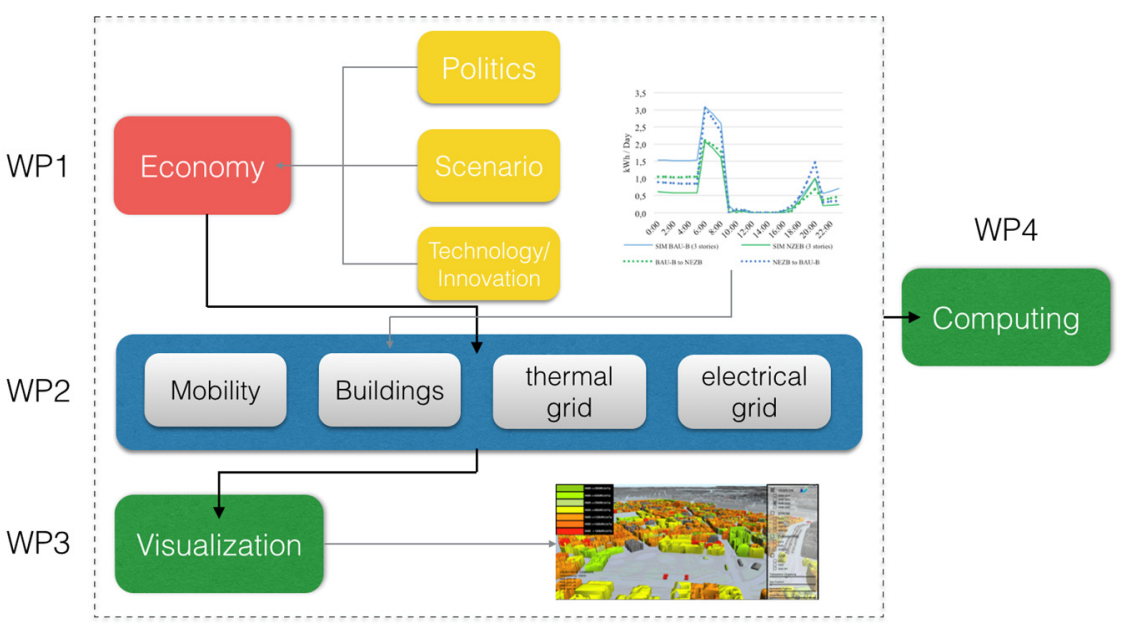

Figure 8: Workflow within the URBEM.

Figure 9 presents an example result of the URBEM [3] prototype and indicates a time snapshot of some parts of the fourth district. The different colours of those buildings are representing the differences of the thermal quality in terms of heating demand for this particular time step or even in one year balance based on density functions which were discussed in the paper.

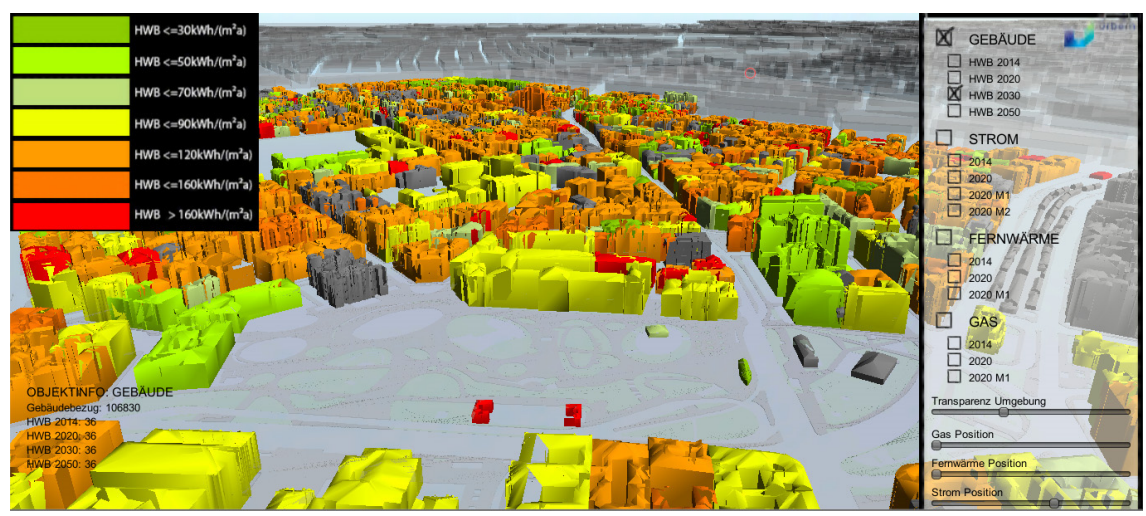

Figure 9: URBEM Prototype of the visualization of buildings regarding the annual heating demand for the 4th district in Vienna. 
Based on figure 9, the results for the energy distribution, e.g. district heating grid, are getting visualized in a similar way. This way to visual results is highly promising to support decision makers and stakeholders for future decisions towards a sustainable, affordable and liveable city.

\section{Conclusion and outlook}

This paper demonstrated a new approach on how to implement the discipline building simulation within a newly developed and comprehensive simulation environment. URBEM deals with future challenges in terms of urbanization and sustainable cities. This tool is going to enable a better understanding regarding the entire energy flow within a city with respect to real characteristics of all buildings, the entire energy distribution and generation, economics, lifestyle aspects and visualization. Furthermore it supports decisions makers such as the city council or of the utility company.

The results for all investigated density functions are sufficiently accurate for using those generated heating load profiles within the URBEM simulation environment. The simulation efforts within the considered boundaries (e.g. district or city level) by using density function instead of individual commercial building simulation software for each building gets minimalized at an equal and accurate level of quality. Furthermore initial parameters such as the annual heating or cooling demand, construction year, type of building or building use case are sufficient to get the right density function for the right scenario within the URBEM simulation environment. Further research in this field will be to extend the density functions on cooling demand, HVAC-Systems for balancing at the level of Et [10], electrical load profiles, more building types, improved performance during spring and aunt and a variation of different lifestyle aspects.

Further research within the URBEM will be the continuous work, the simulation interoperability for different simulation fields and to enable automatically requesting of initial parameters to run the entire simulation. Due to URBEM is not done yet, more results and research activities will be published soon.

\section{Acknowledgements}

The author acknowledges the Wiener Stadtwerke and the Vienna University of Technology for funding this research in the framework of a $\mathrm{PhD}$ within the URBEM. Furthermore the author acknowledges the supervisor of his $\mathrm{PhD}$ work.

\section{References}

[1] Vienna University of Technology, https://www.tuwien.ac.at/en/tuwien_home/

[2] Wiener Stadtwerke, http://www.wienerstadtwerke.at/ 
[3] URBEM - urban energy and mobility system. (Vienna University of. Technology, Editor) http://urbem.tuwien.ac.at/home/EN/

[4] Crawley et al., Contrasting the capabilities of building energy performance simulation programs. Proc. of the Building Simulation Conference, Montreal: IBPSA 2005.

[5] Maile et al., Building Energy Performance Simulation Tools - a Life Cycle and Interoperable Perspective. Center for Integrated Facility Engineering, Stanford 2007.

[6] Ziegler et al., Validated Load Profiles In Terms Of Density Functions For Residential And Non-Residential Buildings In Order To Enhance The Simulation Capability In A Comprehensive Urban Simulation Environment. Proc. of the International Building Physics Conference, Turin: IBPC 2015.

[7] OiB. Teil6 - Energieeinsparung und Wärmeschutz. Vienna: Österreichisches Institut für Bautechnik, 2011.

[8] Woloszyn et al., Annex 41: Whole Building Heat, Air and Moisture Response: Modelling Principles and Common Exercises. Executive Committee on Energy, Conservation in Buildings and Community Systems: IEA - International Energy Agency 2008.

[9] Korenjic et al., An analytical solution of a moisture transfer problem for coupled room and building component. Energy and Buildings 2011.

[10] Yoshino et al., Annex 53: Total Energy Use in Buildings: Analysis \& Evaluation Methods. Energy in Buildings and Communities Programme: IEA - International Energy Agency 2013. 